\title{
Evaluación de dos marcas comerciales de pruebas de ELISA para el diagnóstico de HTLV-1 frente a muestras peruanas
}

\section{Evaluation of two brands of test of ELISA for the diagnosis of HTLV-1 against Peruvian samples}

\author{
Emilio Tasayco-Magallanes', Eduardo Miranda-Ulloa', Soledad Romero-Ruiz', Fany Cárdenas-Bustamante', \\ Ronal Briceño-Espinoza ${ }^{1}$ y Benedicta Yana-Calatayud ${ }^{1}$
}

'Laboratorio de Referencia Nacional Virus de Transmisión Sexual VIH/SIDA, Centro Nacional de Salud Pública, Instituto Nacional de Salud. Lima, Perú.

Financiamiento: Co-financiado por el tesista y por el Instituto Nacional de Salud de Perú.

Los autores declaran no tener conflictos de interés en la publicación de este artículo.

Recibido: 5 de marzo de 2020 / Aceptado: 20 de noviembre de 2020

\section{Resumen}

Por ser el Perú un país endémico de HTLV-1, es importante conocer la confiabilidad de las marcas comerciales para el diagnóstico más usadas en nuestro país. En el presente estudio, evaluamos dos marcas comerciales de pruebas de ELISA (Biokit y Wantai) para la detección de anticuerpos contra HTLV-1 frente a muestras peruanas. Ambas marcas comerciales fueron evaluadas frente a 242 sueros: 123 positivos a HTLV-1 y 119 negativos a HTLV-1 (referencia: inmunoblot). Se estimaron los parámetros de precisión diagnóstica. La sensibilidad, especificidad, VPP, VPN e índice de validez de Biokit fueron: $100 \%$, $93,2 \%, 93,8 \%, 100 \%, 96,6 \%$; respectivamente y de Wantai fue de $99,1 \%$ para todos los parámetros. Concluimos, que la marca Biokit fue la más adecuada para ser usada en el Laboratorio de Referencia Nacional de Virus de Transmisión Sexual, VIH/SIDA de Perú.

Palabras clave: HTLV-1; ELISA; Biokit; Wantai.

\section{Introducción}

S e estima que en el mundo existen entre 10 y 20 millones de personas infectadas con el virus linfotrópico de células $\mathrm{T}$ humanas tipo 1 (HTLV-1), de las cuales entre 2 a 2,5 millones corresponden a Brasil y entre 150.000 y 450.000 a Perú ${ }^{1}$.

El Perú es endémico a la infección por HTLV-1 y el principal causante de la leucemia-linfoma de células $\mathrm{T}$ del adulto y de la paraparesia espástica tropical ${ }^{2}$. Entre 5 y $10 \%$ de los infectados desarrollan las enfermedades aso-

\begin{abstract}
As Peru is endemic to HTLV-1, it is important to know the reliability of the most used screening trademarks in our country. In the present study, we evaluated two brands of ELISA (Biokit and Wantai) for the detection of antibodies against HTLV-1/2 against Peruvian samples. Both brands were evaluated against 242 sera: 123 positive for HTLV-1 and 119 negative for HTLV-1 (Reference: Immunoblot). We estimated diagnostic accuracy parameters. The sensitivity, specificity, PPV, NPV and validity index of Biokit were: 100\%, 93.2\%, $93.8 \%, 100 \%, 96.6 \%$; respectively and Wantai were $99.1 \%$ for all parameters. We conclude that the Biokit brand is the most suitable for use in the National Reference Laboratory of Sexually Transmitted Viruses HIV/AIDS, Peru.
\end{abstract}

Keywords: HTLV-1; ELISA; Biokit; Wantai. 
pruebas de ELISA, donde las marcas comerciales Biokit (sensibilidad: 100\% y especificidad: 99,8 a $100 \%)^{7}$ y Wantai (sensibilidad: 99,9 a $100, \%$ y especificidad: 99,8 a $100, \%)^{8}$ son las más utilizadas y se encuentran disponibles en el mercado nacional.

El rendimiento diagnóstico de cada marca comercial de pruebas de ELISA está referido, por lo general, al país del fabricante o donde se realizó la evaluación. Sin embargo, lo ideal es medir su rendimiento utilizando muestras del país donde se utilizan los kits y así obtener datos más cercanos a la realidad, permitiendo recomendar su uso o rechazarlos. Las muestras reactivas a las pruebas de ELISA son confirmadas mediante inmunofluorescencia indirecta, inmunoblot y RPC anidada múltiple ${ }^{9,10,11}$.

Por lo descrito, se propuso como objetivo evaluar la reactividad de dos marcas comerciales de ELISA (Biokit y Wantai) para la detección de anticuerpos contra HTLV-1 frente a muestras peruanas.

\section{Método}

Durante el año 2019, se realizó un estudio observacional prospectivo de evaluación de una prueba diagnóstica, cuyo protocolo fue aprobado por el Comité Institucional de Ética en Investigación del Instituto Nacional de Salud (INS) de Perú. Se evaluaron 242 sueros procedentes de la seroteca de
HTLV, del Laboratorio de Referencia Nacional de Virus de Transmisión Sexual VIH/SIDA (LRN VTS VIH/SIDA) del INS de Perú. De los cuales, 123 fueron positivos y 119 negativos a HTLV-1, siendo inmunoblot la prueba de referencia (Fujirebio, Bélgica. "INNO-LIA HTLV I/II"; sensibilidad: $100 \%$, especificidad: 99 a 99,7\%; capacidad de diferenciación: $98,8 \%)^{10}$. El tamaño de la muestra (242 sueros), que fue por conveniencia, correspondió al total de las muestras ingresadas al INS para su confirmación por HTLV entre el 1 de enero y el 30 de junio del 2017.

Todas las muestras fueron procesadas por las marcas: Biokit Bioelisa HTLV-1/2 5.0 $0^{7}$ y ELISA Wantai HTLV-1/2 ${ }^{8}$. Los ensayos fueron realizados de acuerdo a las instrucciones del fabricante (inserto).

El análisis estadístico se realizó usando el programa Epidat v3.1 y Excel; se calculó la sensibilidad, especificidad, índice de validez, valor predictivo positivo (VPP), valor predictivo negativo (VPN) e índice de Youden, considerando un nivel de confianza de 95\%.

\section{Resultados}

La marca Biokit no obtuvo ningún falso negativo, sin embargo, dio ocho falsos positivos; mientras que la marca Wantai dio un falso positivo y un falso negativo (Figura 1).

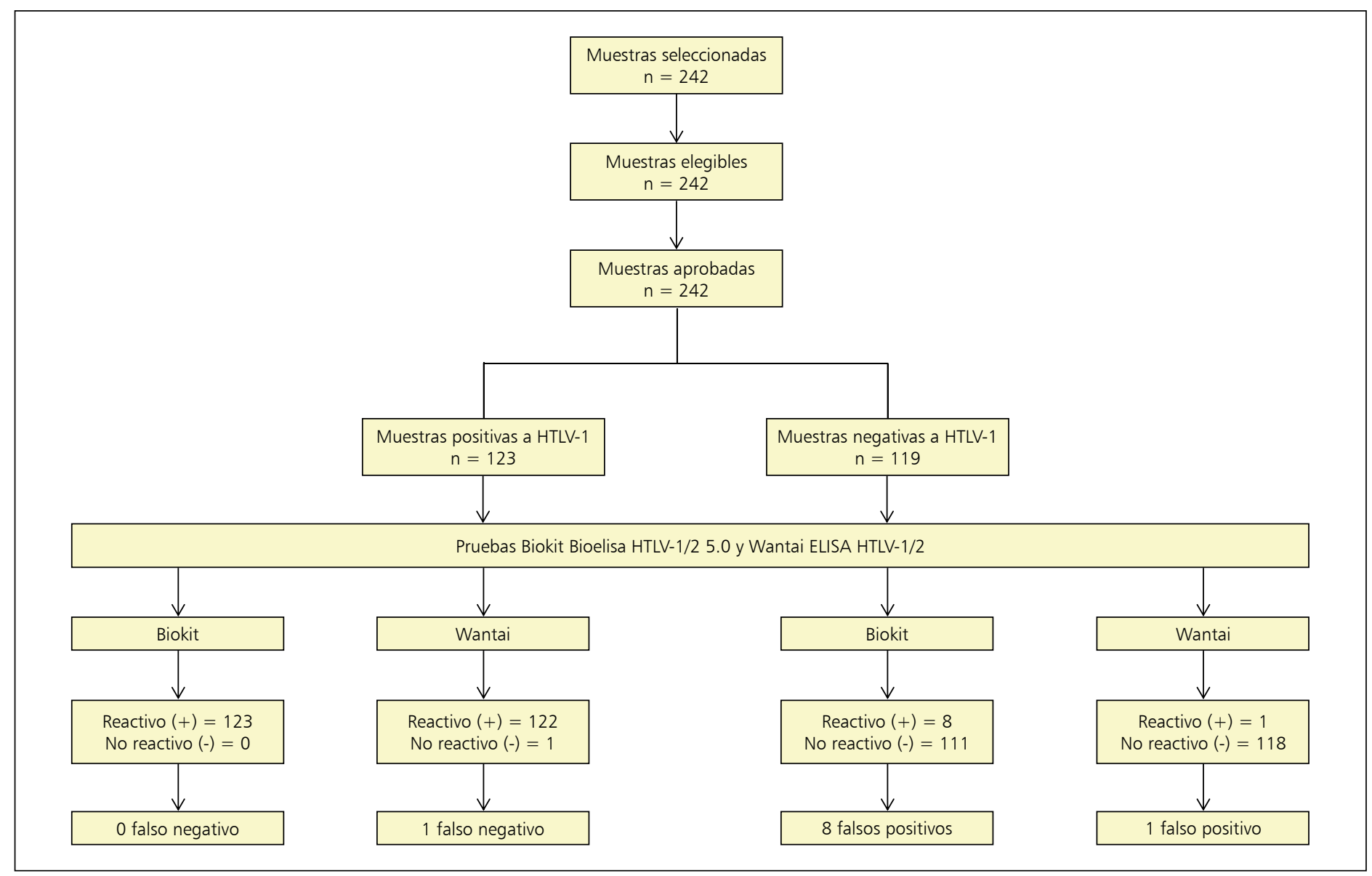

Figura 1. Flujo de muestras incorporadas al estudio. 
Tabla 1. Resultados de los parámetros evaluados de las pruebas ELISA Biokit y ELISA Wantai para la detección de anticuerpos contra HTLV-1

\begin{tabular}{lcr}
\hline Parámetros & ELISA Biokit & ELISA Wantai \\
& Valor (IC95\%) & Valor (IC95\%)
\end{tabular}

\begin{tabular}{lrlrc}
\hline Sensibilidad (\%) & 100,00 & $(99,59-100,00)$ & 99,19 & $(97,19-100,00)$ \\
\hline Especificidad (\%) & 93,28 & $(88,36-98,20)$ & 99,16 & $(97,10-100,00)$ \\
\hline Índice de validez (\%) & 96,69 & $(94,24-99,15)$ & 99,17 & $(97,83-100,00)$ \\
\hline Valor predictivo + (\%) & 93,89 & $(89,41-98,38)$ & 99,19 & $(97,19-100,00)$ \\
Valor predictivo - (\%) & 100,00 & $(99,55-100,00)$ & 99,16 & $(97,10-100,00)$ \\
Índice de Youden & 0,93 & $(0,89-0,98)$ & 0,98 & $(0,96-1,0)$ \\
\hline
\end{tabular}

IC95\%: Intervalo de confianza al 95\% hace deducir que lo ideal es realizar evaluaciones frente a muestras del país donde se usan los kits y se obtengan valores más cercanos a cada realidad.

Una de las limitaciones de nuestro estudio fue que la seroteca de HTLV del INS de Perú cuenta sólo con escasos sueros positivos a HTLV-2, y por ello sólo se evaluó frente a sueros positivos a HTLV-1; esta baja disponibilidad se debe a que el Perú no es endémico a HTLV-2. Otra limitación fue que no se evaluaron otras marcas de ELISA de menor uso.

La marca con mejor rendimiento diagnóstico fue Biokit Bioelisa, por cumplir con los criterios de una prueba de cribado, obteniendo entre sus resultados la mayor sensibilidad y una aceptable especificidad. Asimismo, de acuerdo a los resultados obtenidos, concluimos que el reactivo más adecuado para ser usado en el LRN VTS VIH/SIDA-INS como prueba de cribado para detectar anticuerpos contra HTLV-1 es Biokit Bioelisa, por lo que recomendamos su uso de manera prioritaria. En caso de no contar con la disponibilidad de ésta, se debe usar como segunda opción la marca ELISA Wantai.

\section{Referencias bibliográficas}

fue superior a Wantai y que la especificidad y el índice de validez de la marca Wantai, fue superior a la de Biokit. Asimismo, el índice de Youden obtenido, 0,93 y 0,98 para Biokit y Wantai; respectivamente, nos confirmó que la posibilidad de tener falsos positivos o negativos es baja (Tabla 1).

\section{Discusión}

Los resultados obtenidos de sensibilidad muestran una similitud a lo reportado en los insertos de cada marca evaluada (Biokit: España y Francia; Wantai: China $)^{7,8}$; Biokit no mostró falsos negativos ( $100 \%$ versus $\left.100 \%{ }^{7}\right)$, a diferencia de Wantai que sí tuvo un falso negativo (99,1 versus $99,9 \%{ }^{8}$ ). En cuanto a la especificidad, nuestro resultado de Wantai fue comparable a lo reportado en su inserto $\left(99,1 \%\right.$ versus $\left.99,8 \%{ }^{8}\right)$; sin embargo, con Biokit sí se observó cierta diferencia $\left(93,2 \%\right.$ versus $\left.99,8 \%{ }^{7}\right)$. A pesar de ello, su utilidad no se vería afectada por ser una prueba de cribado. Siendo las pruebas de ELISA utilizadas como cribado, lo ideal es que presenten la máxima sensibilidad (100\%) para no tener falsos negativos y dejar de diagnosticar individuos infectantes. Por ello algunos reportes recomiendan informar en el resultado del paciente, el porcentaje de la sensibilidad de las pruebas ${ }^{12-14}$.

Hace una década, una investigación peruana evaluó otras marcas de ELISA (Platelia, Murex y Ortho) ${ }^{15}$. Estos investigadores concluyeron y recomendaron que para realizar el descarte de la infección por HTLV-1, se utilice una combinación de al menos dos marcas comerciales de ELISA antes de considerarlos negativas. Aquellas marcas no alcanzaban el 100\% de sensibilidad, posiblemente debido a que usaban antígenos nativos de la lisis viral (primera generación) ${ }^{15}$, a diferencia de los actuales que usan antígenos recombinantes ${ }^{7,8}$. Si se comparan los resultados combinando las dos marcas que se evaluaron, se alcanzaría una sensibilidad global de $100 \%$ y una especificidad global de $99,1 \%$; la cual la hace comparable a la prueba de referencia usada.

Un estudio realizado en Argentina reportó una sensibilidad de 99,7\% y una especificidad de $97 \%$ para la marca Biokit ${ }^{16}$; otro estudio realizado en Brasil reportó $100 \%$ de sensibilidad y $100 \%$ de especificad para la marca Wantai $^{8,13}$. Al comparar dichos resultados con los nuestros, observamos una diferencia en el parámetro de la sensibilidad para ambas marcas, lo cual nos
1.- Gessain A, Cassar O. Epidemiological aspects and world distribution of HTLV-1 infection. Front Microbiol 2012; 3: 388. doi: 10.3389/ fmicb.2012.00388.

2.- Ita F, Mayer E F, Verdonck K, González E, Clark D, Gotuzzo E. Human T-lymphotropic virus type 1 infection is frequent in rural communities of the southern Andes of Peru. Int J Infect Dis 2014; 19: 46-52. doi: 10.1016/j. ijid.2013.10.005.

3.- Gotuzzo Herencia E, González Lagos E, Verdonck Bosteels K, Mayer Arispe E, Ita Nagy F, Clark Leza D. Veinte años de investigación sobre HTLV-1 y sus complicaciones médicas en el Perú: Perspectivas generales. Acta Méd Peruana 2010; 27: 196-203.

4.- Instituto de Salud Pública de Chile. Virus linfotrópico de células T humano tipos I y II (HTLV-I/II). Chile, 2010-2014. Boletín Instituto de Salud Pública de Chile, 2015; 5(8): [aprox. 7 p.]. Disponible en: http://www.ispch. cl/sites/default/files/BOLETIN\%20HTLV.pdf. Fecha de acceso: 6 de febrero 2020.

5.- El Peruano. Decreto Supremo que declara de interés nacional la realización del Congreso Mundial sobre la Infección por el Virus Linfotrópico de Células T Humanas - HTLV-I/II. Decreto supremo N 028-2018-SA. https:// busquedas.elperuano.pe/download/url/decreto-supremo-que-declara-deinteres-nacional-la-realizaci-decreto-supremo-n-028-2018-sa-1712240-2. Fecha de acceso: 6 de febrero de 2020]. Italia: DiaSorin S.p.A.; 2012 Disponible en: http://www.annardx.com/ productos/images/productos/diagnostica/infecciosas/liaison-xl-murex-rechtlv-i-ii-310270.pdf. Fecha de acceso: 6 de febrero 2020.

7.- Biokit. Bioelisa HTLV-I+II 5.0 Product improvement [Internet]. Barcelona, España: BIOKIT, S.A.; 2010 Disponible en: file:///C:/Users/emiranda/ Downloads/3000-1165_Package_Insert_PT.pdf https://www.biokit.com/ bioelisa-htlv-iii-50. Fecha de acceso: 6 de febrero de 2020.

8.- HTLV Antibody Diagnostic ELISA Kit. [Inserto]. Wantai. Beijing, China. 2013. Disponible en: https://es.scribd.com/document/397644565/WantaiHtlv-Elisa\#from_embed. Fecha de acceso: 6 de febrero de 2020.

9.- Romero-Ruiz S, Miranda-Ulloa E, Briceño-Espinoza R. Rendimiento diagnóstico de la prueba de inmunofluorescencia indirecta para la detección de anticuerpos contra HTLV-1. Rev Peru Med Exp Salud Pública 2017; 34 (3): 461-5. doi: 10.17843/rpmesp.2017.343.2635.

10.- INNO-LIA ${ }^{\mathrm{TM}}$ HTLV I/II Score. [Inserto]. Fujirebio Technologiepark. Belgium. 2011. Disponible en: http://www.bio-protech.com.tw/databank/ DataSheet/PathoDiag/Innogenetics/user's\%20manual/80540_HIV_
6.- DiaSorin. LIAISON ${ }^{\circledR}$ XL MUREX recHTLV-I/II (REF 310270). Saluggia, 


\section{Comunicación Breve}

Score_110704.pdf. Fecha de acceso: 6 de febrero de 2020.

11.- Serrano K. Estandarización y validación de la técnica de Reacción en Cadena de la Polimerasa Nested Múltiple para el diagnóstico de HTLV-1. [tesis]. Universidad Nacional de Trujillo. Trujillo, Perú, 2019. Disponible en: http://dspace.unitru.edu.pe/bitstream/handle/ UNITRU/12575/SERRANO\%20SEGURA\%2c\%20Kevin\%20Osnar. pdf? sequence=1\&isAllowed=y. Fecha de acceso: 6 de febrero de 2020 .

12.- Moreno C, Balangero M, Barbás MG, Cudolá A, Gallego S. Diagnóstico serológico de HTLV-1/2: combinación de técnicas de tamizaje para definir el estatus serológico en donantes de sangre. Rev Argent Microbiol 2013; 45 : 165-8. doi: 10.1016/S0325-7541(13)70019-1

13.- Ministério da Saúde. Guia de manejo clínico da infecção pelo HTLV. Brasília-2013. Disponible en: http://www.sierj.org.br/artigos/htlv_manual_ final_pdf_25082.pdf. Fecha de acceso: 6 de febrero de 2020.
14.- Ministerio de Salud. Gobierno de Chile. Protocolo de atención de pacientes con HTLV-I. 2018 - 2 ${ }^{\mathrm{a}}$ versión. Disponible en: https://diprece.minsal.cl/ wp-content/uploads/2019/01/2019.01.18_PROTOCOLO-HTLV.pdf. Fecha de acceso: 6 de febrero 2020.

15.- Verdonck K, González E, Maldonado F, Agapito D, Van Dooren S, Vandamme A M, et al. Comparison of three ELISAs for the routine diagnosis of human T-lymphotropic virus infection in a high-prevalence setting in Peru. Trans R Soc Trop Med Hyg 2009; 103: 420-2. doi: 10.1016/j.trstmh.2008.12.002.

16.- Berini C A, Pascuccio M S, Bautista C T, Gendler S A, Eirin M E, Rodríguez C, et al. Comparison of four commercial screening assays for the diagnosis of human T-cell Lymphotropic virus types 1 and 2 . J Virol Methods. 2008; 147 (2): 322-7. doi: 10.1016 / j.jviromet.2007.09.012. 